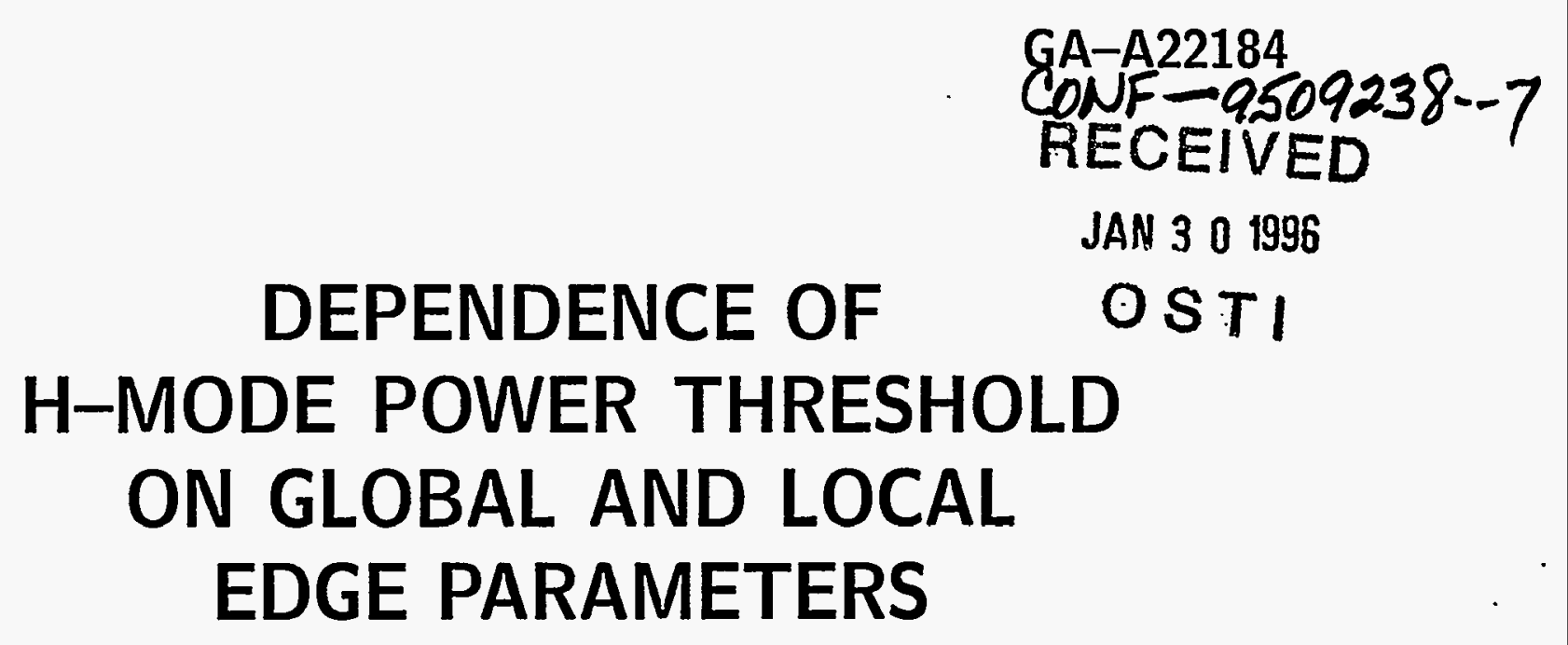

by

R.J. GROEBNER, T.N. CARLSTROM, K.H. BURRELL,

P. GOHIL, J. KIM, and G.M. STAEBLER

DECEMBER 1995 


\section{DISCLAIMER}

This report was prepared as an account of work sponsored by an agency of the United States Government. Neither the United States Government nor any agency thereof, nor any of their employees, makes any warranty, express or implied, or assumes any legal liability or responsibility for the accuracy, completeness, or usefulness of any information, apparatus, product, or process disclosed, or represents that its use would not infringe privately owned rights. Reference herein to any specific commercial product, process, or service by trade name, trademark, manufacturer, or otherwise, does not necessarily constitute or imply its endorsement, recommendation, or favoring by the United States Government or any agency thereof. The views and opinions of authors expressed herein do not necessarily state or reflect those of the United States Government or any agency thereof. 


\title{
DEPENDENCE OF \\ H-MODE POWER THRESHOLD \\ ON GLOBAL AND LOCAL \\ EDGE PARAMETERS
}

by

R.J. GROEBNER, T.N. CARLSTROM, K.H. BURRELL,

P. GOHIL, J. KIM, and G.M. STAEBLER

This is a preprint of a paper presented at the Fifth Workshop on H-Mode Physics, September 18-20, 1995, Princeton, New Jersey, and to be printed in the Proceedings.

\author{
Work supported by \\ U.S. Department of Energy \\ Contract DE-AC03-89ER51114
}

GENERAL ATOMICS PROJECT 3466 DECEMBER 1995

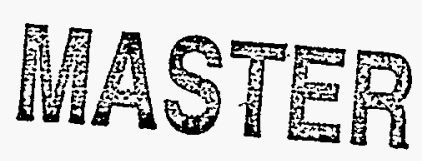




\title{
Dependence of $\mathrm{H}$-mode power threshold on global and local edge parameters
}

\author{
R J Groebner, T N Carlstrom, K H Burrell, P Gohil, J Kim \\ and $\mathbf{G} \mathbf{M}$ Staebler
}

General Atomics, San Diego, California, USA

\begin{abstract}
Measurements of local electron density $n_{e}$, electron temperature $T_{e}$, and ion temperature $T_{\mathrm{i}}$ have been made at the very edge of the plasma just prior to the transition into $\mathrm{H}$-mode for four different single parameter scans in the DIII-D tokamak. The means and standard deviations of $n_{e}$, $T_{e}$, and $T_{i}$ under these conditions for a value of the normalized toroidal flux of 0.98 are respectively, $1.5 \pm 0.7 \times 10^{19} \mathrm{~m}^{-3}, 0.051 \pm 0.016 \mathrm{keV}$, and $0.14 \pm 0.03 \mathrm{keV}$. The threshold condition for the transition is more sensitive to temperature than to density. The data indicate that the dependence is not as simple as a requirement for a fixed value of the ion collisionality.
\end{abstract}

Keywords. Fusion; tokamak; confinement; H-mode; L-mode;

I to H transition; DIII-D

\section{Introduction}

H-mode research during the last few years has produced several theories of the transition to H-mode (see review by Itoh and Itoh, 1995). At the level of testing which has been performed so $\mathrm{far}$, these theories are generally in qualitative agreement with experiment (Groebner, 1993). However, quantitative comparisons between theory and experiment are few (Ida et al., 1992; Burrell et al., 1992). If progress is to be made in using theory to develop predictive models for the transition to $\mathrm{H}$-mode, it is necessary that much more detailed quantitative theory-experiment comparisons be performed. One important part of such comparisons is to experimentally determine the conditions at the edge of the plasma which allow the plasma to evolve into H-mode. This paper summarizes initial work along these lines which has been performed as part of $\mathrm{H}$-mode threshold studies in the DIII-D tokamak.

The strategy used for this work is to measure the electron density $n_{\mathrm{e}}$, electron temperature $T_{e}$, and ion temperature $T_{i}$ at the edge of the plasma just prior to transition to H-mode. The data reported here have been obtained from a series of single parameter scans used to determine the $\mathrm{H}$-mode power threshold under various conditions. These scans include a scan of the lineaveraged electron density, a scan of the toroidal magnetic field $B_{\mathrm{T}}$, a scan of the plasma current $I_{\mathrm{p}}$, and a scan of the gap between the two separatrices for double null discharges. Nearly all edge parameters observed in these scans fall into the ranges of $n_{\mathrm{e}} \approx 0.3$ to $3.5 \times 10^{19} \mathrm{~m}^{-3}, T_{\mathrm{e}} \approx 25$ to $100 \mathrm{eV}$, and $T_{\mathrm{i}} \approx 100$ to $200 \mathrm{eV}$. With the possible exception of the separatrix scan, little or no variation of edge temperature (ion or electron) was observed within a scan. In general, the results suggest that the transition depends more sensitively on edge temperature than on edge density.

\section{Experimental Method}

The goal of this study is to determine the values of $n_{\mathrm{e}}, T_{\mathrm{e}}$, and $T_{\mathrm{i}}$ at the last closed flux surface (LCFS) which are required for the transition to $\mathrm{H}$-mode to occur. The choice of the LCFS as the location for the measurements is based on the premise that the $\mathrm{L}-\mathrm{H}$ transition is triggered very close to the LCFS. The results reported here have been obtained from discharges used to determine the scaling of the H-mode power threshold with global parameters. The 
general technique has been discussed previously (Carlstrom, 1994). Briefly, the neutral beam heating power was increased in small steps until a transition to H-mode was observed. The edge parameters in this study are then evaluated just prior to the transition.

The edge values of $n_{e}$ and $T_{e}$ were measured with a multipoint, multipulse Thomson Scattering system. This system has a high density of edge chords, which provide a spatial resolution of $0.75 \mathrm{~cm}$ when projected to the midplane of the vessel. In addition, this system uses eight YAG lasers controlled by a flexible timing system which provided data every 5 to $25 \mathrm{~ms}$ for the discharges discussed here. The edge values of $T_{i}$ were measured with a multipoint Charge Exchange Recombination (CER) system which also has a high density of edge chords, with a spatial resolution capability of $0.3 \mathrm{~cm}$. For most of the data presented here, the spatial resolution is $0.6 \mathrm{~cm}$. This system is designed for fast readout of the detector and has a minimum temporal resolution of $0.5 \mathrm{~ms}$. The coarsest temporal resolution used for this study is $20 \mathrm{~ms}$. The data for the CER system were obtained from the C VI $5290 \AA$ line excited by charge transfer between one of the heating neutral beams and fully ionized carbon ions in the plasma.

\section{Results}

Except as noted, the standard discharge for the results presented here was a single null diverted discharge with the grad-B drift in the direction of the $\mathrm{X}$-point. The plasma current was 1.0 to $1.35 \mathrm{MA}$, the toroidal field was $2.1 \mathrm{~T}$, and deuterium neutral beams were injected into a deuterium plasma. About this standard set of conditions, single parameter scans were done in which the toroidal field, the plasma current and the line-averaged electron density were separately varied. Also, results are presented from a series of double null discharges in which the magnetic equilibrium was adjusted slightly so that discharges varied from a configuration in which the power flow was primarily to the $\mathrm{X}$-point in the direction of the grad-B drift to a configuration in which the power flow was primarily to the opposite null.

Local values of $n_{e}, T_{e}$, and $T_{i}$ will be presented for values of the reduced toroical magnetic flux (labeled here as $\rho$ ) of 0.95 and 0.98 . A more correct approach might be to evaluate the data at $\rho=1.00$ (the value of $\rho$ at the LCFS). However, the data set is more complete for $\rho=0.98$ than for $\rho=1.00$. The values observed at $\rho=0.98$ are about $0.5 \mathrm{~cm}$ from the LCFS and are sufficiently close to those at $\rho=1.00$ that it is believed that they provide an adequate view of the edge for the purposes of this paper. All of the data were obtained within less than $20 \mathrm{~ms}$ prior to the transition. Due to the fact that these discharges were operated near the power threshold, the edge parameters were not changing rapidly prior to the transition and there is little error from using data within 10 to $20 \mathrm{~ms}$ before the transition.

The primary sources of uncertainty in the data derive from various systematic issues rather than statistical errors. These systematic errors include an uncertainty in the mapping to $\rho$ of about \pm 0.02 , dithering in some discharges and possibly fluctuations in the data due to turbulence. It is crudely estimated that errors of up to $30 \%$ to $40 \%$ could arise from these systematic effects.

In the toroidal field scan, the power threshold increased linearly with $B_{\mathrm{T}}$ as $B_{\mathrm{T}}$ varied from 1.0 to $2.1 \mathrm{~T}$. In contrast, figure 1 shows no significant change of edge $n_{e}, T_{e}$, or $T_{i}$ during this scan. In the plasma current scan, the power threshold increased weakly with $I_{\mathrm{p}}$ as $I_{\mathrm{p}}$ varied from 1.0 to $2.0 \mathrm{MA}$. Figure 2 shows little if any significant change of edge $n_{e}, T_{e}$, or $T_{\mathfrak{i}}$ during this scan. In the scan of line averaged electron density, the power threshold increased (less strongly than linearly) as the density was raised from 1.2 to $4.0 \times 10^{19} \mathrm{~m}^{-3}$. As figure 3 shows, the edge density increased by at least a factor of four in this scan but edge temperatures were essentially unchanged. Figure 4 shows data from a series of double null discharges in which the distance between the two separatrix surfaces, as measured at the midplane, was varied. As this distance $D r_{\text {sep }}$ was varied from -0.6 to $0.5 \mathrm{~cm}$, the threshold power varied from 4 to $14 \mathrm{MW}$. This modification of the magnetic topology changed the power outflow from the plasma from being predominantly in the grad-B drift direction to the opposite direction. As shown in figure 4 , all edge parameters tended to increase as $D r_{\text {sep }}$ became more positive. 


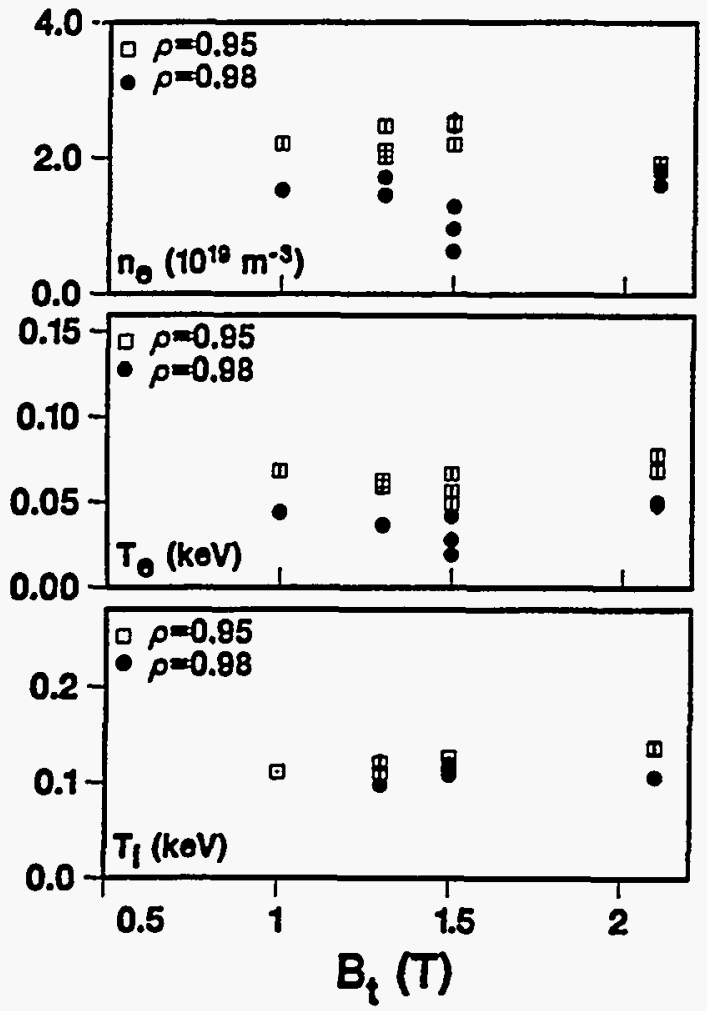

Figure 1. Values of $n_{e}, T_{e}$, and $T_{j}$ near LCFS during variation of toroidal magnetic field. Single null discharge $(\mathrm{SND}), I_{\mathrm{p}}=1.0 \mathrm{MA}, \mathrm{D}^{0}$ beams injected into $\mathrm{D}^{+}$plasma.

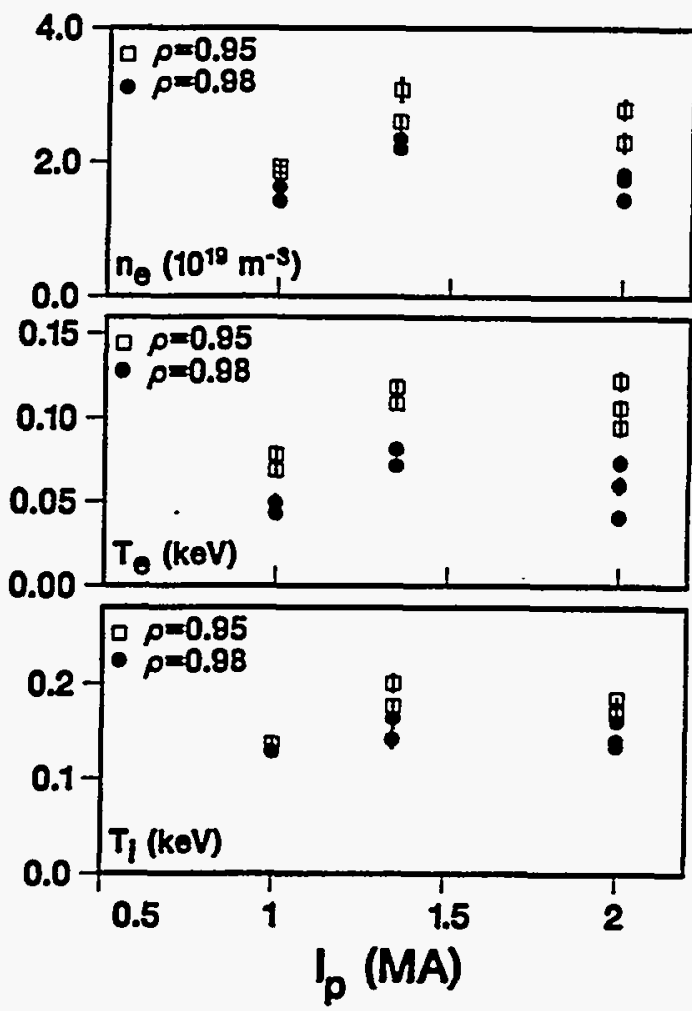

Figure 2. Values of $n_{e}, T_{e}$, and $T_{1}$ near LCFS during variation of plasma current. SND dis charge, $B_{\mathrm{T}}=2.1 \mathrm{MA}, n_{\mathrm{e}}=4 \times 10^{19} \mathrm{~m}^{-3}, \mathrm{D}^{0}$ beams injected into $\mathrm{D}^{+}$plasma.

\section{Discussion and Conclusions}

The simplest way to characterize all of the results presented in figures 1 through 4 is to examine the mean and standard deviation of these data. At $\rho=0.98$ (within less than $1 \mathrm{~cm}$ of the plasma edge), the mean and standard deviations for $n_{e}, T_{e}$, and $T_{i}$, respectively, are $1.5 \pm 0.7 \times 10^{19} \mathrm{~m}^{-3}, 0.051 \pm 0.016 \mathrm{keV}$, and $0.14 \pm 0.03 \mathrm{keV}$. When expressed as fractions of the means, the standard deviations for $n_{e}, T_{e}$, and $T_{i}$ are, respectively, $47 \%, 30 \%$, and $22 \%$. These results indicate that the transition occurs within a narrower range of temperature than of density and thus suggest that the trigger condition for the transition is a more sensitive function of temperature (ion or electron) than of density. This conclusion is consistent with previous work which indicates that sufficient edge temperature is necessary to trigger the transition to H-mode (ASDEX, 1989; Groebner et al., 1992).

The density scan (shown in figure 3 ) can be used to examine the dependence of the transition on the ion collisionality parameter $\nu_{i \star}$ which is proportional to $n_{\mathrm{i}} / T_{\mathrm{i}}^{2}$ where $n_{\mathrm{i}}$ is the ion density. Under the assumption that $n_{\mathrm{i}}$ is proportional to $n_{\mathrm{e}}$ for the density scan (that $Z_{\text {eff }}$ is constant), the data of figure 4 imply a variation of $\nu_{i \star}$ of 5.4. This result indicates that the threshold condition is not as simple as achieving some value of edge $\nu_{i \star}$, such as $\nu_{i \star}=1$. The only way to avoid this conclusion is to postulate that the edge $n_{\mathrm{i}}$ actually varied little during the density scan. This seems unlikely but direct measurements of edge $n_{i}$ or $Z_{\text {eff }}$ are not available.

In conclusion, for a wide range of discharges, the transition to $\mathrm{H}$-mode occurs for a fairly well defined range of temperatures, particularly of ion temperature. The threshold condition does not appear to be as simple as a fixed value of edge $\nu_{i \star}$. Further work is required to ameliorate 


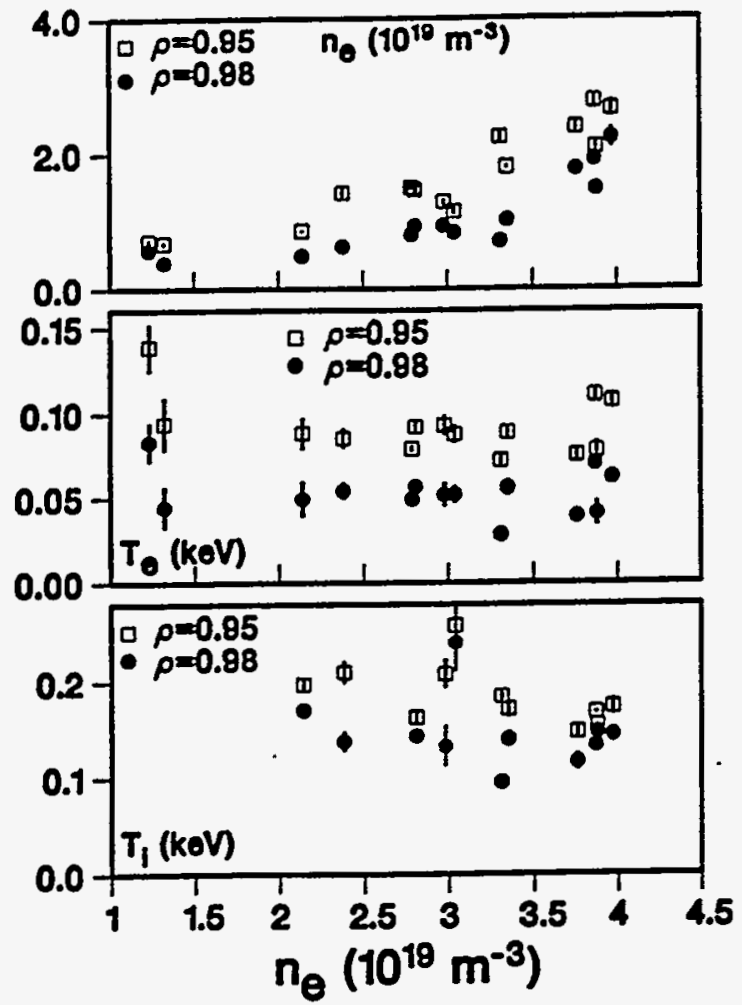

Figure 3. Values of $n_{e}, T_{e}$, and $T_{i}$ near LCFS during variation of line averaged electron density. SND discharge, $I_{p}=1.35 \mathrm{MA}$, $B_{\mathrm{T}}=2.1 \mathrm{MA}, \mathrm{D}^{\circ}$ beams injected into $\mathrm{D}^{+}$ plasma.

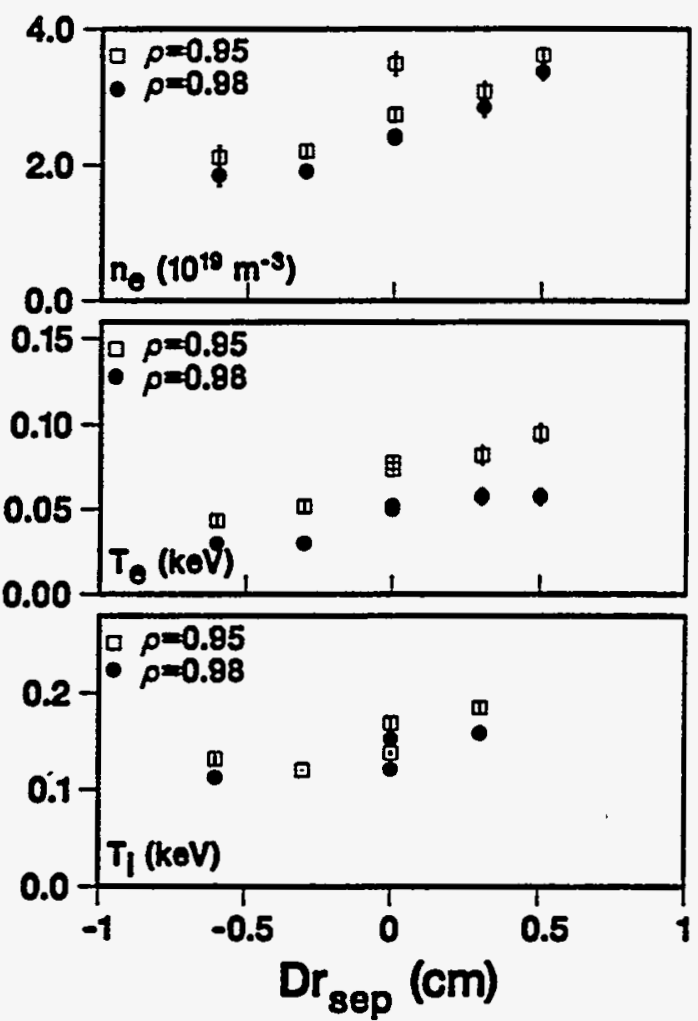

Figure 4. Values of $n_{e}, T_{e}$, and $T_{i}$ near LCFS during variation of gap between two separatrix surfaces in double null discharge. $I_{p}$ $=1.35 \mathrm{MA}, B_{\mathrm{T}}=2.1 \mathrm{MA}, n_{\mathrm{e}}=4 \times 10^{19} \mathrm{~m}^{-3}$, $\mathrm{D}^{0}$ beams injected into $\mathrm{D}^{+}$plasma.

the impact of the systematic effects, discussed above, to extend this research to additional single parameter scans, to add additional edge parameters, and to search for a function of edge parameters which unifies the results from all of the scans.

\section{Acknowledgment}

This is a report of work sponsored by the U.S. Department of Energy under Contract No. DE-AC03-89ER51114.

\section{References}

ASDEX Team 1989 Nucl. Fusion 291959

Burrell $\mathrm{K} H$ et al. 1992 Plasma Phys. and Contr. Fusion 341859

Carlstrom T N et al. 1992 Plasma Phys. and Contr. Fusion 36 A147

Groebner R J, Burrell K H, Gohil P, Kim Y B and Seraydarian R P 1992 Proc. 19th European

Conf. on Controlled Fusion and Plasma Physics Innsbruck, v 16C, p 183

Groebner R J 1993 Phys. Fluids B 52343

Ida K et al. 1992 Phys. Fluids B 42552

Itoh K and Itoh S-I "The Role of Electric Field in Confinement," to be published in Plasma Phys. and Contr. Fusion 
